\title{
Hybrid simulation modelling of the human-production process interface in lean manufacturing systems
}

\author{
Omogbai Oleghe, Konstantinos Salonitis
}

\begin{abstract}
Purpose - This study seeks to advance a system dynamics-discrete event hybrid simulation modelling concept useful for taking improvement decisions where one needs to consider the interactions between human factors and process flow elements in lean manufacturing systems.
\end{abstract}

Design/methodology/approach - A unique approach is taken to hybrid simulation modelling where the whole problem situation is first conceptualized using a causal loop diagram and a stock and flow diagram, before transmitting to a hybrid simulation model. The concept is intended to simplify the simulation modelling process and make the concept pliable for use in various types of lean manufacturing problem situations.

Findings - The hybrid simulation modelling concept was applied to a lean manufacturing case where quality performance was sporadic mainly because of production pressures. The hybrid modelling concept revealed a solution that advanced full compliance with lean and one that required changes in job scheduling policies in order to promote both continuous improvement and throughput increases.

Research implications - Because non-tangible aspects of lean were objectively assessed using the hybrid modelling concept, the study is an advancement towards establishing a credible link between human resource aspects of lean and the performance of an organization.

Practical implications - The applied hybrid model enabled managers in the plant navigate the trade-off decision they often face when choosing to advance production output ahead of continuous improvement practices.

Originality/value - System dynamics-discrete event hybrid simulation modelling is a rarity in in lean manufacturing systems.

Keywords - Lean manufacturing system; System dynamics; Discrete event simulation; Hybrid simulation modelling

Paper type Research paper

\section{Introduction}

Lean consists of a collection of continuous improvement practices intended to banish waste and create value for the customer (Womack et al., 1990; Liker, 2004). Implementing, sustaining and improving lean is sometimes challenging as there are multiple issues that can derail its performance. For example, when companies are affected financially, they could decide to cut maintenance budget and so the performance of Total Productive Maintenance (TPM) practices may decrease. Sometimes safe working practices are skipped when there is production pressure, because employees place greater emphasis on production than on safety (Probst and Graso, 2013). Training and upskilling of workers is important for lean to thrive and grow, yet it is among the first items discarded when companies are cutting cost (Owens, 2006; Sung and Choi, 2014). These and many other issues are real, some are subtle such as worker attitude and others may be dormant until external factors cause them to be active. Lean manufacturing managers should be well equipped with tools that will enable them navigate those problems that may derail lean at any 
time.

The investigation of lean often warrants using simulation modelling (Robinson et al., 2012; Omogbai and Salonitis, 2016). System dynamics (SD) and discrete event simulation (DES) are two established simulation modelling (SM) methodologies, and both have been widely applied to investigate the performance of lean practices in manufacturing system. A system dynamicsdiscrete (SD-DES) hybrid simulation model is a unique mixed-method simulation model. It is unique in the sense that the SD and DES sub-models interact (exchanging data), while both submodels are run as a single model. The SD and DES sub-models in the hybrid are intended to mimic different aspects of the system being investigated. In real-life, these aspects may be continuously and discretely interacting while affecting each other. Hence in the hybrid, both SD and DES must interact and exchange data (Morgan et al., 2017). For example, materials planning decisions are taken at an enterprise level, whereas these decisions are based on shop-level scheduling of the materials (Venkateswaran and Son, 2005; Rabelo et al., 2015). A hybrid that is needed to model this type of system would require that the SD sub-model (enterprise level decision making) and the DES sub-model (shopfloor decision making) interact to mimic the reallife situation where they are dependent on each other to evaluate the plan at discrete time steps (Venkateswaran and Son, 2005)

Lean as it relates to manufacturing systems, includes concepts that have both tangible and intangible variables (Oleghe and Salonitis, 2015). Take Kanban for example. The size of kanban bins can be quantified precisely and even optimized. However, decisions taken or managements' attitude towards the size of the bins is something that cannot be precisely measured or quantified. Moreover, it may change depending on conditions within the system, such as resource or material availability. Hao and Shen (2008) in fact make firm submissions that a DES-only analysis of Kanban systems is too simplistic, yet DES continues to be the tool of first choice for modellers when simulating Kanban. Whereas, Rabelo et al. (2015) assert that SM analysis of modern systems should be "approached by methods that are able to accommodate the continuous and discrete modes of behaviour". Lean concepts, including those relating to manufacturing systems, are sociotechnical in nature and they involve interactions between people and the systems they operate in (Hadid et al., 2016). Investigating lean manufacturing (LM) systems on the basis of people and systems, as well as the decisions people take, may require mixing simulation methods if SM is intended to be used (Tekippe and Krejci, 2016).

SD-DES hybrid simulation models have been advanced in health care (Brailsford et al., 2010; Chahal et al., 2013; Dierks et al., 2008; Tejada et al., 2014), construction (Alvanchi et al., 2011; Alzraiee et al., 2012) and even in traditional manufacturing systems (Helal, 2008; Rabelo et al., 2015). Morgan et al. (2017) and Barbosa and Azevedo (2017) have provided the most up-to-date review of studies where SD-DES hybrid modelling methods were used. They did not reference any study that was related to a LM system. In addition, we did not come across the use of a SDDES hybrid simulation model in a LM system setting. The use of single-method SM is what is prevalent, giving one the impression that there is a research gap. The only hybrid we found was for Agent Based Modelling and DES for a kanban-based material handling system (Hao and Shen, 2008). So, even if a simulation researcher needed to apply a SD-DES hybrid SM concept to a LM system case study, there are no foundational studies he/she can build on. The simulation researcher would have to rely on what has been developed in other non-LM system settings, the concepts of which may be incompatible with those of a LM system.

The current work is motivated partly by the paucity of SD-DES hybrid SM methods in LM systems, and partly by the need to solve a real-life problem where a SD-DES hybrid simulation model needed to be used. The aim of this study is to advance a SD-DES hybrid SM concept for modelling the human-production process interface of LM systems. The intention is to advance a tool with which managers can use to investigate deeply those issues that cause or may cause lean 
to detail at any time. A tool to enable them navigate the trade-off decision they often face when choosing to advance production output ahead of continuous improvement practices, and so address the sporadic nature of lean in their plant. The study is also intended to generate interest in the use of SD-DES hybrid modelling in LM systems. The type of hybrid SM is something that is needed if one were to go by the variety of problems often encountered in LM systems where single-method simulation modelling would prove incapable of addressing. If the analysis of other systems such as healthcare, construction and even traditional manufacturing systems have all necessitated the use of SD-DES hybrid modelling, why not LM systems which present similar problem situations. Unfortunately, the SD-DES hybrid modelling frameworks that have been advanced previously by others are not so easy to replicate in situations other than where they were promoted because of their problem/context-specific approach (Chahal et al., 2013). Most attempts seem to have been ad-hoc with no clear methodology (Eldabi et al., 2016). To this end, the remainder of this document is structured as follows: section two provides background information about the LM system case; in section three a SD-DES hybrid SM concept is designed, presented and applied to the case; section four discusses the academic and practical implications of the study while section five concludes with future outlook.

\section{The case study notes}

The case of in-line, along the process quality checks for a print packaging manufacturer is presented. The presented problem fitted one where both SD and DES needed to be used, in a simultaneous and hybrid fashion. Supposedly, the company has implemented LM concepts, yet defect rate remains high and fluctuates widely. Multiple issues relate to defect rates such as machine malfunctioning, raw materials quality, worker experience level and many others. In the current study, the searchlight was beamed on quality checks as it was an area that could be investigated and improved in the short run. By focusing on only one aspect at a time, the eventual hybrid model is less likely to be complex and easier for stakeholders to comprehend than where multiple issues are included in a large model.

The following notes were taken during a 2-day assessment of the plant. Interviews with key stakeholders and participants in the system, sifting through archival records, and generally observing the system are ways one can use to glean information that would be used to build a simulation model (Chahal et al., 2013; Robinson et al., 2010; Sterman, 2000). The first day involved just observing the system, the work flow and the work practices. With the information gathered during the first visit, we were able to start the second day with a list of questions to ask key participants in the plant including machine operators, supervisors and managers. Interviews included the use of open ended questions, in an unstructured manner, mostly done while the operator was operating the machine or when the manager was on the shopfloor monitoring the factory. It is at this stage that we began to build a mental map of the problem and how simulation would be used to address the problem.

Web type of printing is done in the plant. The machines are semi-automated and visual quality checks are necessary as the web film is being processed (moving through) on the machine. For example, with the web printing machine, web moving out of alignment occurs randomly and if not detected on time will progressively worsen and generate defective printed materials. Also, if the ink viscosity is not checked for consistency, it can create a lighter or incorrect shade of ink on the printed material and this adds to the defects. Due to the nature of the machines, these visual and manual checks need to be performed regularly and meticulously as the web material is moving, to eliminate all variations that lead to defects. Unless the machines are upgraded to fully automated ones, the manual checks could not be exempted or lessened.

Currently in the case study plant, when the work load increases, many of these checks are either not done or they are done superficially. During our observations of work practices in the plant, 
we found that one quality check took roughly five seconds. This time was insufficient to do a comprehensive quality check. However, it was gathered that when the work load was lighter, the machine operators spent close to eighteen seconds on each quality check and generally carried out more comprehensive checks. We also noticed from plant records that defect rates were considerably lower when work load was reduced in the plant. We could ascribe this behaviour to the number of activities that needed to be concluded within a given period of time, many of which, including quality checks, were competing for operator time and effort. Workers were more concerned with clearing order backlog than on quality performance when the work load increased. Although there were standard operating procedures and checklist documents clearly pasted on each machine, they were not strictly adhered to: the managers were in favour of anything that would increase or appear to increase throughput. The behaviour we noticed in this plant mirrors one that Dierks et al. (2008) studied on how safety controls are bypassed in the interest of maximizing productivity and throughput. Managers should know when to relax the pressure on throughput and targets, as well as pressure from other sources such as from stakeholders (Woods, 2017).

Another noticeable pattern of behaviour was related to the complexity of a print job. Complexity of print is a function of number and type of colours, the width and thickness of the web and the art design. It was found that the higher the complexity of the print job, the higher the defect rate, because the required number of quality checks increased as job complexity increased. It was also observed that for some highly complex print jobs, the number of printing machine operators assigned to print the job was increased while the machine speed was reduced, because these jobs had been known in the past to generate a higher than normal defect rate. By increasing the number of machine operators on the job and by reducing the speed of the machine, the defect rate could be reduced. To the organization, this type of behaviour seemed to address the problem, however, this policy had many flaws. Firstly, it took more time to produce, worsening the backlog situation. Secondly, other jobs suffered because fewer operators were available to effectively process them. In fact, it was observed that the quality checks for the other jobs were not done properly because those machines were under-staffed when complex jobs were being processed on other machines.

In addition to the above, we found that the speed of machines was sometimes increased to try to meet up with an ever-growing backlog of job orders. When machine speed is increased, the effectiveness of visual quality checks is reduced as some defects are not quickly picked up on the web. This further increases the defect rates in the plant.

It would appear from the foregoing reported observations that the defect rate would be fluctuating always and the order backlog will be increasing. This is because there is no attempt to control those issues that cause the system to deteriorate or to be in constant state of flux. It is obvious that there are multiple impediments to quality checks in the system. The simulation-based study documented in the current paper was initiated to investigate the performance of quality checks and seek ways of improving its performance for the plant. Because managers in the plant will be reluctant to implement any changes that do not also improve throughput, the improvements needed to reduce order backlog situation. In other words, the simulation-based study needed to show how to improve defect rate and order backlog simultaneously.

It is worth documenting the data collection protocol that was used in this phase of the study. We needed to be confident that we had collected enough data to enable us conceptualize and build a SD-DES hybrid simulation model. Figure 1 is the self-explanatory schematic of the data collection protocol. It is advantageous to follow a structured data collection format from the start of the simulation study because data collection and analysis is often needed in case study investigation using unstructured, qualitative data collection methods (Yin, 2014). 


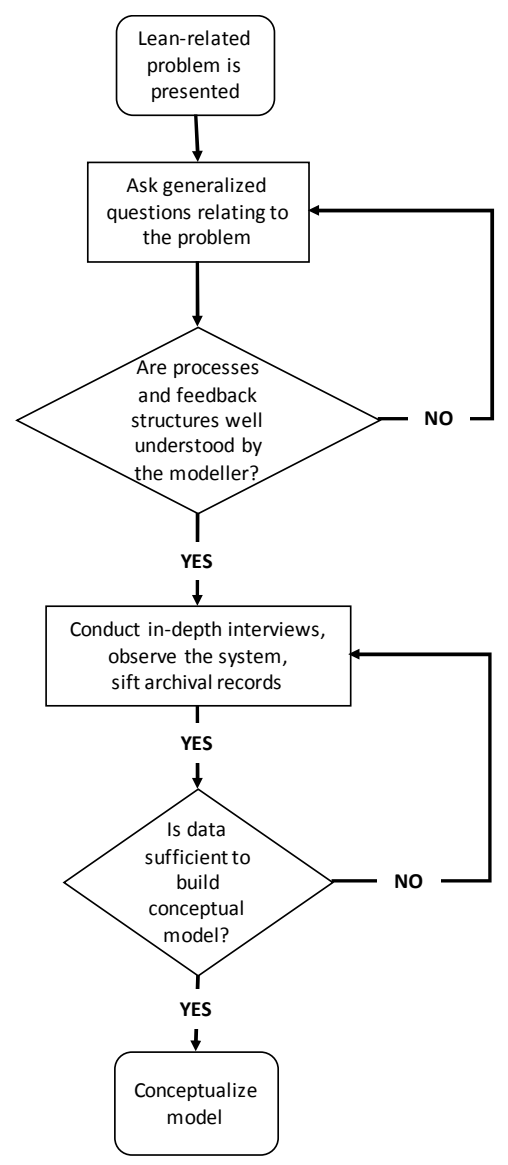

Figure 1. Data collection protocol for first phase of the simulation-based study

\section{Design and application of a system dynamics-discrete event hybrid simulation modelling concept}

Presented in Figure 2 is the conceptual framework that was developed for the SD-DES hybrid SM concept. It is based on the typical path simulation researchers follow when building and using SD, DES and SD-DES hybrid simulation models (Abduaziz et al., 2015; Barton, 2000; Donzelli and Iazeolla, 2001; Alvanchi et al., 2011; Brailsford et al., 2010; Chahal et al., 2013; Zulkepli et al., 2012; Lee et al., 2002; Rabelo et al., 2005; Venkateswaran and Son, 2005; Sterman, 2000; Law and Kelton, 1991). The workflow and features of the individual steps are detailed herewith while demonstrating the concept in the presented case. 


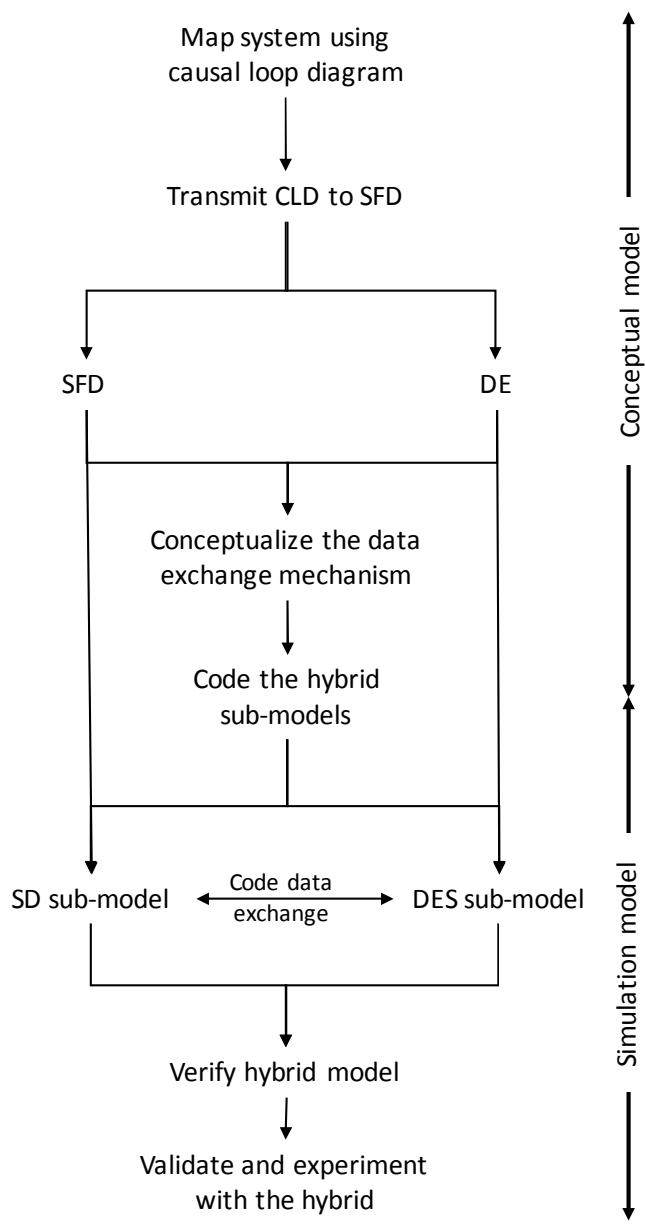

Figure 2. Proposed framework for conducting the SD/DES hybrid simulation modelling study

\subsection{Map system using causal loop diagram}

Robinson et al. (2010) define model conceptualization as "the process of abstracting a model from a real or proposed system" Model conceptualization is akin to a mind map and it is nonsoftware-specific description of the computer simulation model that would be used to investigate the problem (Robinson et al., 2010). The current problem has to do human behaviours (nonphysical concept) which change when there are changes to the conditions in the system they work in. The problem also involves how products (physical entity) are flowed through the system. We propose to use a causal loop diagram (CLD) to map the system and conceptualize a simulation model, because it allows for a holistic view of the system. It captures all or most of the relevant concepts relating to the system or problem situation, including the physical and non-physical concepts all at once. The process of engaging stakeholders at the start (and at other stages) of the simulation modelling study is necessary for ensuring success of the entire process (Robinson et al., 2012; Inam et al., 2015). CLD is more engaging than the other conceptual modelling tools (Jahangirian et al., 2010) and it is effective in capturing the mental models of key stakeholders as well as that of the modeller (Sterman, 2000).

The variables and concepts that are included and mapped in the CLD depend on the system being modelled, the problem that needs to be addressed and the study objectives (Sterman, 2000). 
Simulation models are heavily reliant on logic that is numeric related: concepts and variables for a simulation model should also be empirically quantifiable. Getting consensus and approval of the CLD map and its validity from key stakeholders in the organization is another way of checking that the core variables and concepts have been included, while non-relevant ones have been excluded. Otherwise, the researcher modeller needs to re-navigate the steps shown in Figure 1. CLD is the first major model that a researcher modeller has to present to key stakeholders: it needs to capture their interest to motivate them to carry on with the study.

Investigating and improving the performance of quality checks was the main objective of the simulation-based study. The simulation model would have to include those variables and factors that encourage or prevent workers from consistently carrying out the ideal set of in-process onthe-machine quality checks. We present in Figure 3 the CLD of the problem situation. It is based on the authors' interpretation of the problem as well as the viewpoints of key participants (managers and line supervisors) in the case study system. The important feedback structures have been clearly delineated.

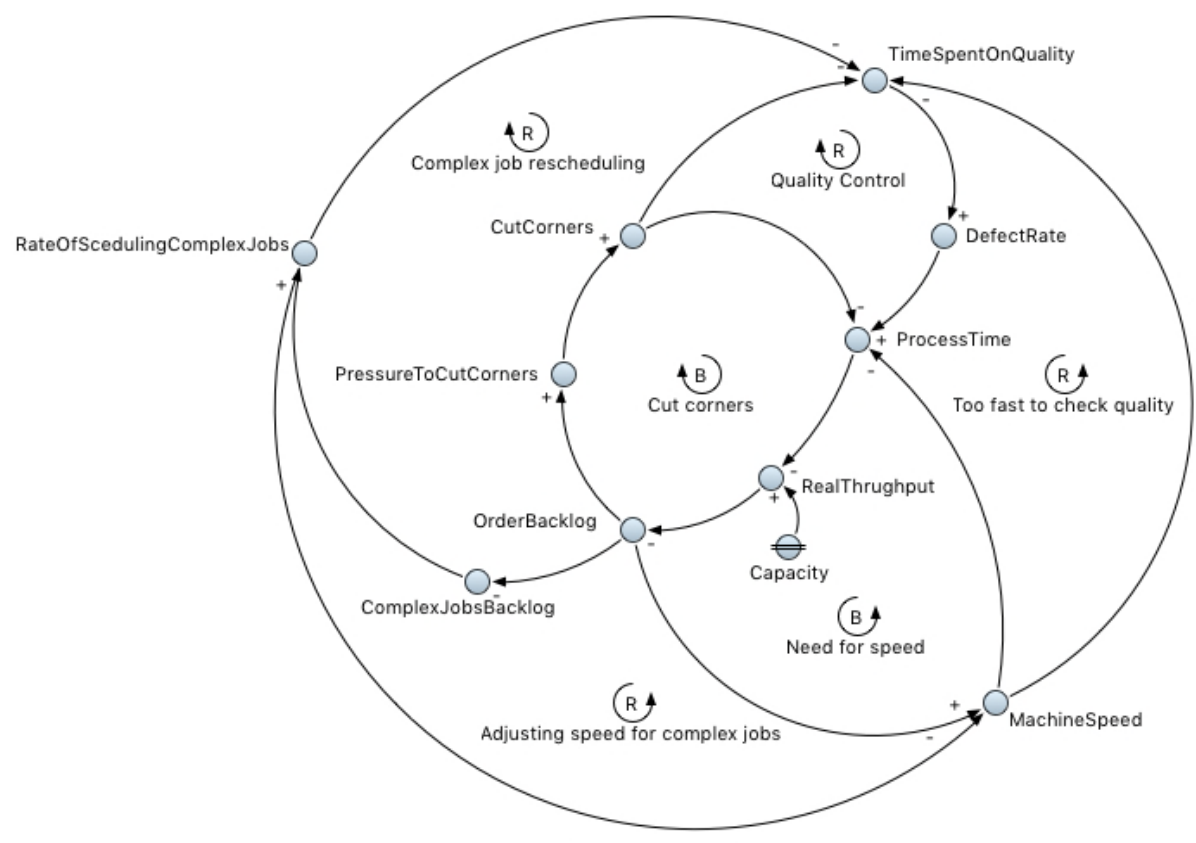

Figure 3. Causal loop diagram of the key variables and concepts relating to the problem situation

\section{Cut corners}

When order backlog increases, it gives the impression that jobs are behind schedule. This increases the work pressure and makes workers to cut corners in order to save time doing tasks. When corners are cut it is intended to save time in doing tasks, so process time reduces and real throughput increases. The increased real throughput makes work flow faster and order backlog to reduce. 
When order backlog increases, the percentage of corners cut by workers increases as machine operators cut corners in tasks, believing this will help reduce the backlog. If number of corners cut increases, the time spent on quality reduces and workers skimp on quality checks. For short term benefits, cutting corners seems to be the better option. If time spent on quality checks reduces, defect detection goes down and defects increases. Defects need to be replaced, this requires re-processing/re-production and reduces the net process time, reducing the real throughput and further increasing the order backlog. The initial state of order backlog is worsened or reinforced.

\section{Need for speed}

When order backlog goes up, workers increase the machine speed in order to reduce process time and so real throughput goes up and backlog reduces. The initial state, like cutting corners, is balanced out.

\section{Too fast to check quality}

When machine speed is increased, rate of detecting defects is reduced because the speed of the web is so fast that it is not easy to detect when there are defects such as web misalignment or colour deviation. Reduction in quality checks adds to worsen the situation by way of defect rates increasing. If defect rate is increased, real throughput is reduced, backlog is increased and machine speed is further increased to improve the situation. The initial state is reinforced.

\section{Scheduling of complex jobs}

When order backlog increases, scheduling of complex jobs rises, in order to reduce total backlog. The more the complex jobs in the system at the same time the higher the defect rate and the lower the real throughput. At lower throughputs, the order backlog increases yet again, reinforcing the initial state. This is a reinforcing feedback loop.

\section{Adjusting speed for complex jobs}

When backlog increases, complex jobs backlog also increases and the rate of scheduling complex jobs increases to reduce the backlog. When complex jobs are being produced, machine speed is reduced. When the number of complex jobs in the system is increased, the net speed losses is increased, worsening the real throughput situation and making order backlog to increase further. The initial situation has worsened in the reinforcing loop.

The naming of feedback loops is simply to label them with terms one can identify with in relation to the problem or system being modelled. Identifying the feedback structures the way we have presented is important when CLD is used to conceptualize a model (Sterman, 2000). In fact, inferences can be made and hypotheses generated based on the feedback loops. If cutting corners and increasing the machine speed marginally improve process time, but have significant negative impact on quality, then cutting corners and increasing machine speed will aggravate the situation. If on the other hand the opposite is the case, then a decision dilemma is faced to either choose to improve throughput over defect rate.

The CLD presented in Figure 3 is a top-level representation, to enable one identify the key feedback structures and conceptualize the simulation-based model. Although mapping the system is non-software specific, we have mapped the problem using AnyLogic software. This is 
intentional as we intended to build both the SD and DES and the eventual hybrid using AnyLogic. It is one of the few simulation software that offer mixed-paradigm modelling.

\subsection{Transmit the CLD to a SFD}

The next step is to transmit the CLD to a stock and flow diagram (SFD), which is a more detailed representation of a CLD, see Figure 4. In a SFD, it is mandatory to include stocks (accumulators of both physical and non-physical items) and flows (which cause the stocks to accumulate or deplete over time) (Sterman, 2000). The dynamics and behaviours in the system revolve around how these stocks increase or decrease over time (Sterman, 2000). The SFD (Figure 4) is an elaborated version of the CLD (Figure 3). It includes variables that can be coded for simulation. It also explains the process and feedback structures in greater detail than the CLD.

In the SFD depiction (Figure 4), one is able to visualize the concepts and variables that may need to be extracted and modelled in DES, for example the process flow concepts or those variables/concepts that illustrate the flow of physical products in the system. In addition, imagining how the CLD will be transcribed to a simulation-based model is another way to decide if a DES is to be used as well, or the extent to which the DES part would be used in the eventual hybrid. For example, the current SFD has been used to model the effect of job scheduling on the performance of workers to undertake ideal quality checks. If the researcher modeller intends to use the eventual simulation model to test different scheduling priority rules, it would make sense to consider using a DES for this part of the problem. This is because it would be programmatically challenging to model priority rules in SD where the stocks and flows hold and move entities on a first-in-first-out basis. A researcher modeller may need to incorporate multiple stocks and flows in SD to mimic detailed priority scheduling. At this detailed level, it becomes expedient to use DES. Moreover, it would be complicated to prioritise or differentiate the flow of discrete entities in $\mathrm{SD}$, whereas, prioritising and scheduling work flow in order to eliminate pressure on quality checks was an improvement that was being considered.

We do not assert that because scheduling is involved that a DES must be incorporated. The study objective is the overall deciding factor, because not all situations may warrant detailed modelling using DES for example, or feedback analysis using SD.

With the above approach where a SFD is modelled first, one might say that the SD-DES hybrid simulation model originates from a SD concept, so it is a SD-dominant approach. It is possible that the DES may at the end dominate the SD part, so there is no saying beforehand if it is a SD or DES dominant hybrid until both sub-models have been conceptualized. What is important is that both SD and DES are needed.

\subsection{Conceptualize the hybrid}

In the current study, the discrete event (DE) part has been used to model the processes and the physical elements relating to the problem. In the intended DE model, the following particulars were of interest:

- Rate of entry of jobs into the system.

- Types/attributes of jobs being entered into the system

- Scheduling rules to prioritise work between the different types of complex jobs and simple jobs

- Processing time for each type of job and its order size 


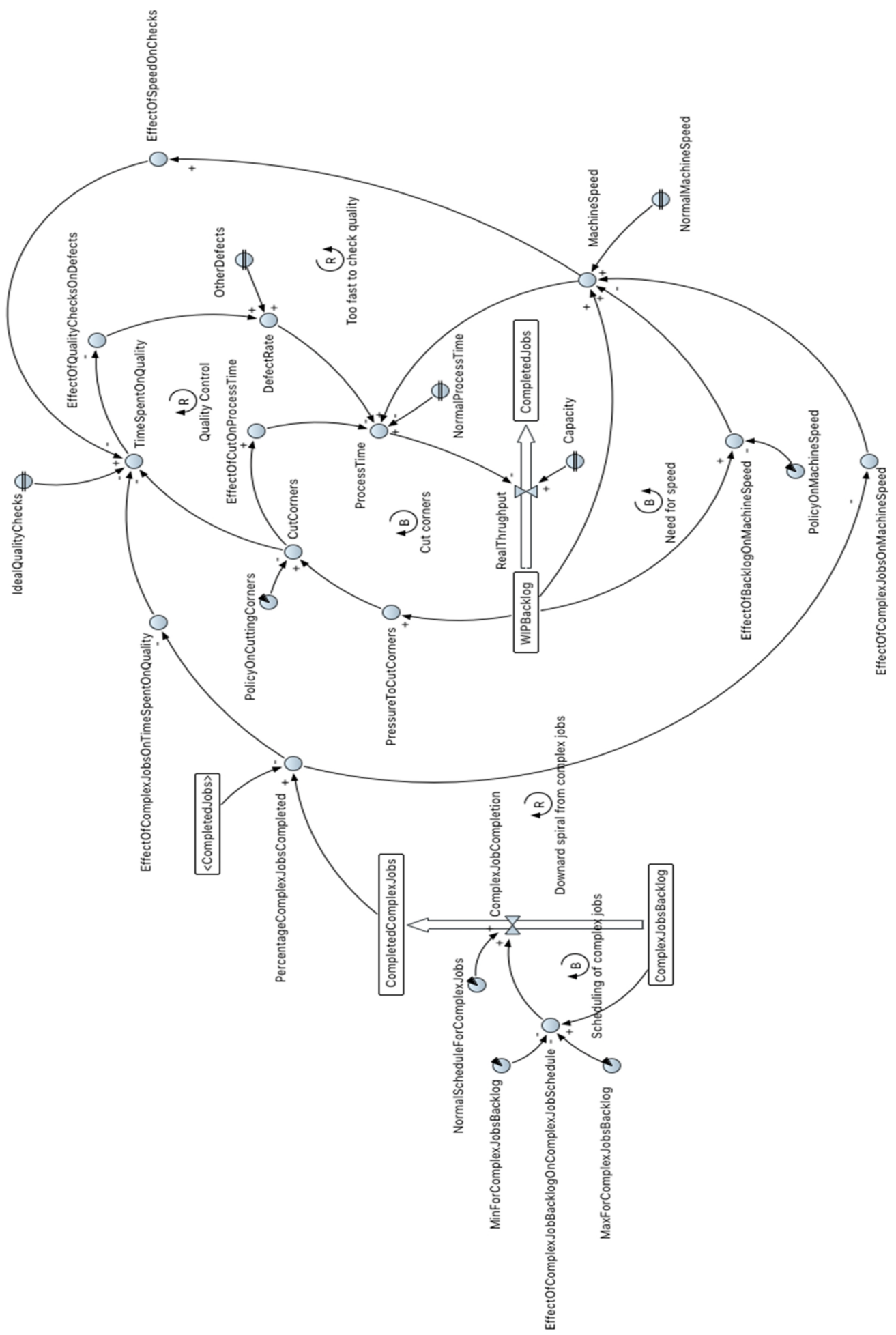

Figure 4. Stock and flow diagram describing the case problem situation

Only the details sufficient to achieve the model objectives have been included (Robinson, 2015). Jobs are entered into the system, they are processed and shipped out. The queue bins have 
been used to represent the backlog for each job type (low complexity, medium complexity, high complexity and simple). The printing machines (five in number) are the only resources being modelled to account for the delays when machines are busy. Jobs are restricted from being processed depending on the scheduling rule to allow complex or simple jobs to be printed or to allow multiple complex jobs to be printed.

The building blocks are adequate for scheduling polices to be tested since scheduling plays an important role in the problem. Although the DE sub-model shown in Figure $\mathbf{5}$ is an exact replica of the intended DES model in the eventual hybrid, the building blocks have not been coded with actual data or logic; they still contain default values at this stage.

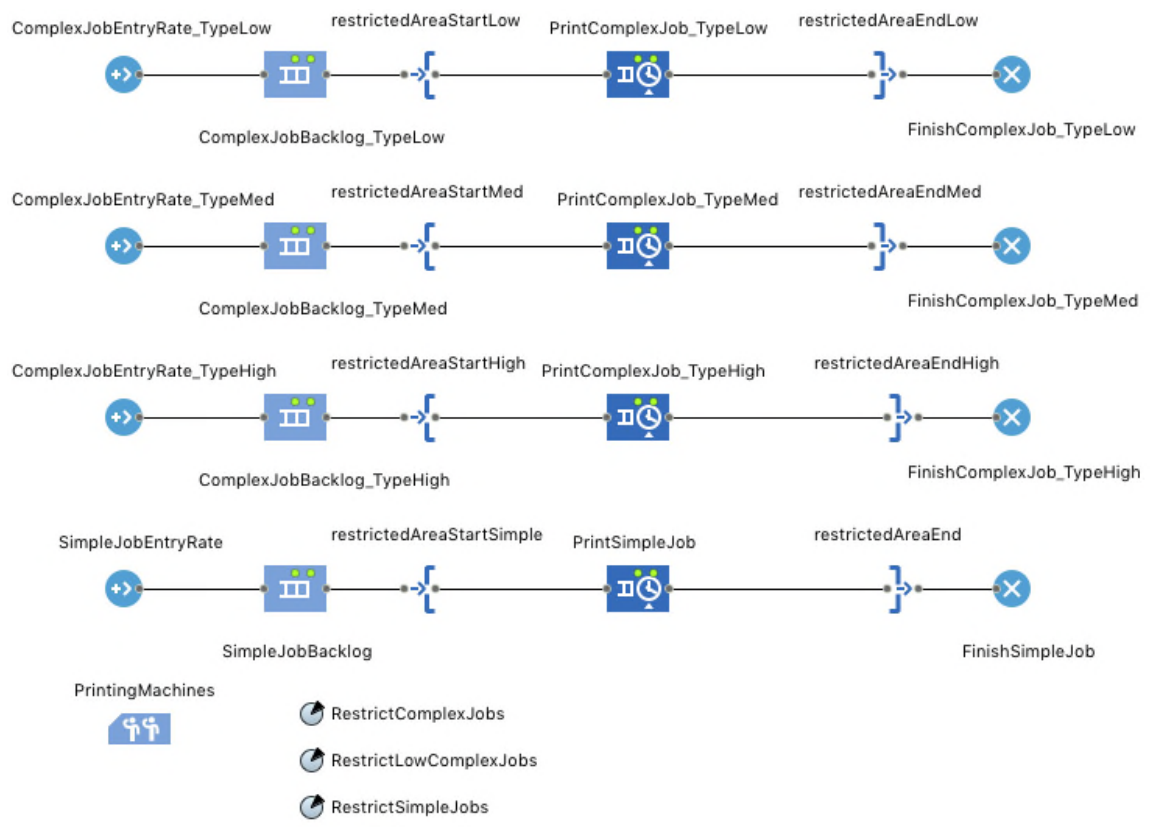

Figure 5. The DE model depicting process for the case study

Because the DE is an extracted model, the SFD (Figure 4) needs to be updated to take account of the concepts and variables that were extracted and modelled using DES. Although stocks and flows are the bedrock of SD modelling, it is possible that some stocks and flows would have been absorbed within the DES to warrant a SD without stocks and flows. For example, real throughput and WIP backlog which were represented as rates and stocks respectively in the initial SFD, have been consequently modelled in the DES flow-and-queue concept. Leaving these stocks and flow rates in the SD sub-model of the eventual hybrid would amount to duplication. The conceptualized hybrid for the current case is presented in Figure 6. It consists of the DE model of Figure 5 and the updated SFD.

The SFD and DE models up till this stage are representing different aspects of the problem. If one were to proceed to simulation at this stage, the approach is a SD-DES mixed-method type. So, a mixed-method SD-DES simulation concept is also possible, but it would not model the interactions between variables that are configured in the different models which is meant to mimic their interactions in the real system. It would only model different aspects of the problem, see for example (Greasley, 2005; Chatha and Weston, 2006; Djanatliev and German, 2013) 


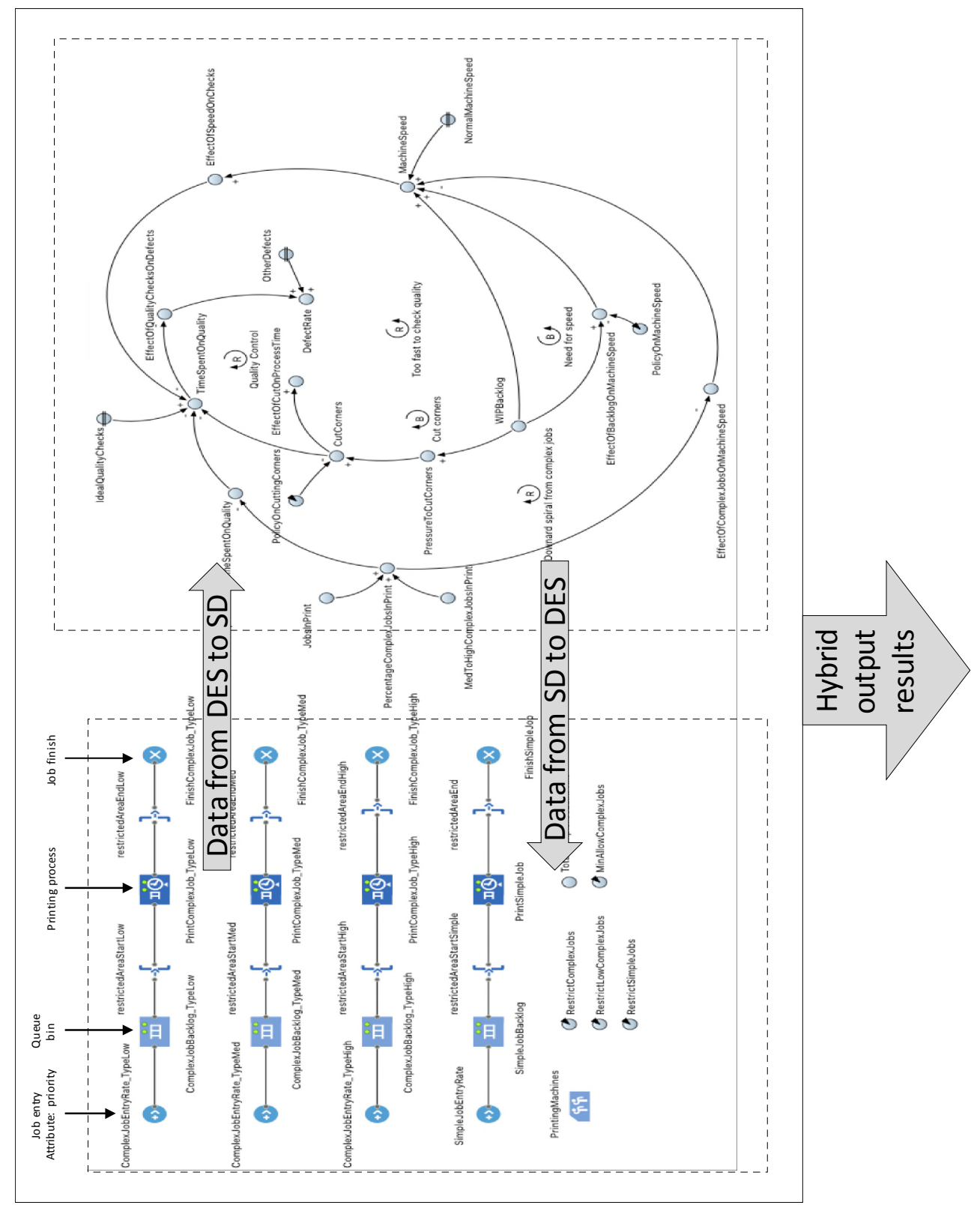

Figure 6. The hybrid SD-DES concept for the case study

\subsection{Conceptualize the data exchange mechanism}

A distinguishing feature with hybrid simulation models is that the sub-models exchange or share data as the hybrid is run. This is intended to mimic real life continuous interaction and influences between different aspects of the system that are being modelled. The literature is replete with different approaches that have been taken to model data exchange between sub-models in the hybrid (Chahal et al., 2013; Alvanchi et al., 2011; Venkateswaran and Son, 2005; Coyle and Wolsterholm, 1980; Rabelo et al., 2015). The choice of method may depend on the skill of the modeller (Lättilä et al., 2010), but one method that has fewer disadvantages and least complex is to use a ready-made hybrid SM toolset (Lättilä et al., 2010; Oliveira et al., 2017) such as AnyLogic and ExtendSim. The number of hybrid simulation researchers using AnyLogic is growing 
(Pruckner and German, 2013; Helal, 2008; Borshchev et al., 2002; Djanatliev and German, 2013; Green et al., 2017). One reason may be that it is one of the few modelling tools that offer multimethod simulation capabilities, which significantly reduces the data exchange complexity in a hybrid. One of the drawbacks is that the modeller needs to be familiar with Java if complex models are to be built, because Java is the programming language for AnyLogic. The models presented in the current case study are small and simple models, so the Java codes needed were not beyond arithmetic and logical expressions.

Because the DE model was extracted from the initial SFD, it is a straightforward task to identify the variables that will exchange data in the eventual hybrid, by simply regarding the DES model as a variable (or sub-model consisting of many variables) within an SD model. In SD, variables affect and are affected by other variables, through the continuous updating of their values, so the DES model is affected and affects other variables in the SD model in the overall hybrid model. Table 1 lists the sender-receiver variables in the intended hybrid model.

Table 1. Summary of conceptualized data exchange operation

\begin{tabular}{|c|c|c|c|c|c|}
\hline Sender & & & Recei & & Updating rate \\
\hline Variable & Model & Type & Variable & Model & \\
\hline $\begin{array}{l}\text { All jobs in printing } \\
\text { process. }\end{array}$ & $\mathrm{DE}$ & Statistics & Jobs in print & SFD & $\begin{array}{l}\text { At the discrete time that } \\
\text { the DE variable changes }\end{array}$ \\
\hline $\begin{array}{l}\text { All complex jobs in } \\
\text { printing process }\end{array}$ & $\mathrm{DE}$ & Statistics & $\begin{array}{l}\text { Medium to } \\
\text { high complex } \\
\text { jobs in print }\end{array}$ & SFD & $\begin{array}{l}\text { At the discrete time that } \\
\text { the DE variable changes }\end{array}$ \\
\hline $\begin{array}{l}\text { Count of all queue } \\
\text { bins }\end{array}$ & $\mathrm{DE}$ & Statistics & WIP backlog & SFD & $\begin{array}{l}\text { At the discrete time that } \\
\text { the DE variable changes }\end{array}$ \\
\hline Machine speed & SFD & $\begin{array}{l}\text { Direct from } \\
\text { variable }\end{array}$ & $\begin{array}{l}\text { Printing } \\
\text { process time }\end{array}$ & $\mathrm{DE}$ & $\begin{array}{l}\text { Continuously as SFD } \\
\text { variable changes }\end{array}$ \\
\hline Defect rate & SFD & $\begin{array}{l}\text { Direct from } \\
\text { variable }\end{array}$ & $\begin{array}{l}\text { Printing } \\
\text { process time }\end{array}$ & $\mathrm{DE}$ & $\begin{array}{l}\text { Continuously as SFD } \\
\text { variable changes }\end{array}$ \\
\hline $\begin{array}{l}\text { Effect of cutting } \\
\text { corners }\end{array}$ & SFD & $\begin{array}{l}\text { Direct from } \\
\text { variable }\end{array}$ & $\begin{array}{l}\text { Printing } \\
\text { process time }\end{array}$ & $\mathrm{DE}$ & $\begin{array}{l}\text { Continuously as SFD } \\
\text { variable changes }\end{array}$ \\
\hline
\end{tabular}

\subsection{Code the SFD and DE sub-models}

Coding the SFD and DE sub-models requires nothing more than inputting factual data of the system being modelled. Factual data is obtained from archival records, work study measurements and observation of the system and behaviours of participants. Sometimes individuals who have expert knowledge about the system may be asked to estimate data, where data does not exist. Coding of the sub-models also includes fitting raw data to known distributions for those that need to be described statistically.

We were not able to collect all the required data during our initial 2-day assessment of the plant. Moreover, the time between our initial assessment of the plant and when the hybrid conceptual model was finalized, was about two weeks. Data requirements for the model had changed from 
when we assessed the plant to when we built the conceptual model. For example, the relationship between WIP backlog and cutting of corners by workers was not an information we singled out to obtain during the assessment of the plant, however after building the conceptual model it was evident that this information was needed.

Companies are not always eager to allow outsiders observe their plant for a study. They are even more sceptical when been asked to provide real production data. It is not surprising because companies like to protect data or information they feel is proprietary. This happened in our case, but by the time we had taken the managers through each of the conceptual models from the CLD to the SFD and DE, and explained what the simulated model could achieve, they changed their stance. When stakeholders are carried along in the simulation study and realize the potential benefits, they are more willing to provide factual data, believing that the study outcomes depend on the reliability of the data. Where data, for example those needed to generate non-linear relationships, is not available or cannot be generated within the time stipulated for the study, the researcher modeller may need to estimate such data. The extant literature holds documented experimental studies. The literature could be searched to reveal if similar studies have been done in the past by other researchers. In fact, Sterman (Sterman, 2000) advises that "omitting structures or variables known to be important because numerical data are unavailable is less scientific and less accurate than using our best of judgement to estimate their value".

\subsubsection{Coding the SFD sub-model}

For the SFD to be coded, two types of data are needed: association data and non-association data. Association data also known as governing equations are those that describe the relationship (linear and non-linear) between a cause and an effect variable. It explains why an effect variable is changing as a result of changes to the cause variable. In CLD and SFD, an arrow originates from a cause variable and links to an effect variable. An example of a governing equation data and how it was derived is described for pressure to cut corners.

We estimated percentage corners cut by observing the time spent on quality checks when the workload is much and comparing this with the ideal time for quality checks. We assume that the effect of order backlog on corner cutting takes an s-shaped curve as it cannot grow indefinitely. For an s-shaped curve, the initial stage of growth is approximately exponential, then as saturation begins, the growth slows, and at maturity, growth stops. Towards the right and left limits of the curve it can be assumed that effects on the response variable do not change significantly. S-shaped curves have applications in many areas. In the case study this s-shaped assumption holds for effect of backlog on corner cutting. As long as backlog is below a certain threshold, corners are not normally cut, in short there is no reason or pressure to cut corners. As backlog increases, the percentage corners that workers are prepared to take increases exponentially. During this phase of the curve, workers believe that significant cut in corners will address the rising order backlog. As they see that backlog is still increasing and their efforts are not yielding the desired effects, they stop increasing their corner cutting measures, but they do not go back to status quo of not cutting corners, convincing themselves that if the corners are not cut, the situation would actually be worse.

For the current case, we estimate this s-shaped function by fitting it to past data on corners cut. The corners cut is maximized at $30 \%$ (or 0.3 ) when order backlog attains a value of 80 job orders and does not grow beyond this. The inflection point is equivalent to 40 job orders. The steepness of the curve was estimated at 0.1 . The equation for this variable is given by Eq. 1 


$$
\text { Pressure to cut corners }=\frac{0.3}{1+e^{-0.1 *(\text { WIPBacklog-40) }}}
$$

Non-association data can be described as those that are used to drive model behaviour. They are represented as constants in variables or in the governing equations. For example, in the Eq. 1, there are three constants $0.3,-0.1$ and 40 which resulted from generating the equation after statistically fitting past data. These values do not change unless they are altered to modify the model behaviour. Another constant is Ideal Quality Checks which was set at 0.3 minutes in the SD model. This represented the ideal time of 18 seconds needed to undertake comprehensive quality checks.

\subsubsection{Coding the DE model}

In the DE model, coding involves inputting mainly non-association type of data. They can also be referred to as constants in the sense that they are used to drive model behaviour. The term constant is not that they do not vary, for example stochastically, but it is that they are not changed unless the model behaviour is to be altered. Logic is also coded into the DE model, for example the priority rules and job routings. The plant keeps records of process time for each job that is processed, i.e. there were archival records we could rely on to code the machine processing time for each job type. In a situation where there are no archival records, and this is very possible in many organizations, the modeller would need to estimate the data based on the judgment of individuals who have expert knowledge about the system. For example, the managers and the machine operators can be asked. Alternatively, the modeller can undertake work studies of the plant, if time permits.

Logic was configured to control the sequencing and scheduling of jobs (including complex jobs) within the model. Priority numbers were assigned to each job type, and logical if-then statements were configured for each job type. The logical statement mimicked the decision to increase the scheduling rate of a job type if its queue count was going above a certain number. We found that this type of logic was easier configured in the DES than if the scheduling part was modelled in detail in SD. So, in a way, the use of the hybrid has also enabled us to simplify programming the model.

\subsubsection{Coding the data exchange}

The approach taken to generate the sender-receiver variable (see Table 1) is more useful when building the sub-models in different software toolsets. When building the hybrid in the same software toolset, coding of the data exchange between the sub-models is straightforward. The modeller simply needs to regard the DES as a variable (or sub-model consisting of many variables) within the $\mathrm{SD}$, with the notion that in $\mathrm{SD}$, variables affect and are affected by other variables, through the continuous updating of their values.

Coding variables to exchange data is on the basis of the information presented in Table 1. Take the first interface in the list between all jobs in printing process in the DES and jobs in print in the SD. The variable "JobsInPrint" in the SD is coded to take the exact values of the total jobs (or items) in the four building blocks representing the printing process in the DES model. The same logic is used for the other two DES to SD interfaces.

In the current case, three interfaces originate from the SD model to the DES model. The initiator variables (defect rate, effect of cutting corners and machine speed) send information to the same 
receiver variable namely printing process. But in setting the delay time in the printing process building blocks, we needed to first input the standard delay time for printing each job type. For printing job types that are of medium complexity, the delay time in the building block PrintComplexJob_TypeMed was configured as triangular $(140,200,180)$. This time represented the standard or normal process time for different categories and order sizes of medium complexity jobs. In the hybrid, the delay time for printing of medium complexity jobs is given by the formula shown in Eq. 2. The delay time is increased by the defect rate because of additional time spent replacing defects. The delay time is decreased by effect of cutting corners and adjustment for machine speed since both are intended to reduce the processing time.

Delay time for Printing Medium Complex Job $=($ triangular $(140,200,180)) *$ (DefectRate - EffectOfCutOnProcessTime - MachineSpeed)

\subsection{Verify the model}

Model verification involves checking that the model has been rightly built. Tests such observing the animation of the model and its outputs are also conducted (Balci, 1998; Greasley, 2005). All abnormalities in model behaviour were corrected at this time. Other tests included checking the structure of the model that it is consistent with the real-life logic it is supposed to mimic, for example checking that decision rules have been captured and functioning well.

\subsection{Validate and experiment with the hybrid simulation model}

\subsection{1. $\quad$ Validate the model}

The model was simulated and the point-by-point results are compared with historical and factual data of the real system. Simulation run time for model use was set at 21,120 minutes ( 2 months $x$ 22 work days per month $\mathrm{x} 480$ minutes per day). It was believed that this was sufficient time for patterns of behaviours to develop and exhibit within the system. Moreover, when orders are piling up in the plant to the point where manufacturing lead times are increasing exponentially, the company would increase capacity by first increasing the man-hours and then include additional shifts. Typically, this coincided with a two-month period after the onset of their highly seasonal demand load. Due to the stochasticity configured in the DES model, it was necessary to do multiple runs. A hundred runs were used with different random seeds and the mean taken (Law and Kelton, 1991; Chongwatpol and Sharda, 2013). The hybrid was run at settings that corresponded with the current situation. The trend in both defect rate and WIP backlog are presented as current as-is, in Figure 7. Validation of the hybrid model was done by comparing the model results with those of the real case. The patterns and trends were also observed as the hybrid model was running. The movements and delays in the DES depicted how jobs are scheduled and processed in the real system. The defect rate variable as well as the backlog in jobs were monitored to validate the model and its behaviour. The current as-is trend as returned by the model depicts what happens in the real system. WIP backlog is always on the rise. Defect rate is low at the start when work load is low, then it rises sharply as work load increases. In addition, defect rate is highly volatile. In the real system, this has been the case as defect rates have been known to follow this trend.

\subsubsection{Experiment with the hybrid simulation model}

The objectives of the simulation-based study determine the types of experiments to be conducted 
with the SD/DES hybrid simulation model. Having prior knowledge about the types of tests that will be conducted with the model is often needed when conceptualizing the simulation model. Parameters and variables can then be included in the model that would be used to conduct these experiments. For example, we intended to test the impact of decisions and policies that permit corners to be cut or machine speed to be increased, because these affected quality control. So, in the SFD concept (see Figure 4) we included two parameter variables namely "Policy on cutting corners" and "Policy on machine speed". The first is a measure of the commitment level to quality. We used a nominal scale to quantify this value. If the policy is one where cutting corners takes precedence over quality checks, a value of 1 is assigned. If the policy is one where quality checks is strictly adhered to no matter the workload condition, a value of 2 is assigned. It is common in the lean literature to measure such qualitative information using nominal and other measurement scale (Goodson, 2002). In the SD model, an if-else logic is used to control this decision. Here we assumed two extremes: it is either there is commitment or not and there are no in-between possibilities or partial commitment. The same was applied to policy on machine speed. If the policy is one where increasing the machine speed is encouraged when backlog begins to rise, a value of 1 is assigned, otherwise a value of 2 is assigned to mimic where machine speed is not increased no matter the backlog situation. It is also possible that other experiments would be revealed, after running some initial ones, for example to gain a better understanding of why the system behaves the way it does for certain scenarios.

In the DES, we included parameters that can be used to modify the way jobs are restricted in the model to mimic different scheduling options. For example, the parameter "Restrict complex jobs" (see Figure 5) is configured to enable us modify the number of jobs complex jobs that can be entered at any given time into the model. In the hybrid model, quality-checks is the only nonconstant variable directly affecting defect rate. On the basis of this, the trend in defect rate will be used as the measure of improvement or deterioration in quality checks. We first set out to establish the effect that cutting corners and increasing machine speed both have on defect rate. To find this out using the model, three behavioural changes are tested with the model:

- Experiment 1- disallow cutting of corners, but allow machine speed

- Experiment 2- disallow machine speed to be increased, but allow corners to be cut

- Experiment 3- disallow both corner cutting and machine speed increase.

One may argue that the above simulation experiments were judgmentally set, based on the study objectives and on the authors' understanding of the system. A Design of Experiments (DOE) approach may have been used in determining an optimal priority setting for jobs in the system. However, using a DOE will add complexity to the simulation study process. Moreover, the hybrid model may not generate "the" precise optimal setting, because some of the variables included in the hybrid model (the SD part) are based on judgmental estimates, for example Eq. 1.

Presenting and rationalizing the simulation result is common practice in research. The experimental results are presented in Figure 7. It is obvious from Figure7a that the backlog situation is the same no matter the decisions that are taken. It means also that there is no gain or loss in throughput. For experiments 1 and 2, the results are likely because the gains expected from cutting corners or increasing the machine speed have been nullified by the defects created as a side-effect. Replacing the defects has taken up production time, and has affected throughput. The result in experiment 3 is indicative that gains in throughput cannot be achieved even by eliminating those things that appear to cause it to diminish. Gains in throughput need to be sought elsewhere, such as in the job scheduling side. As should be expected, defect rate is reduced (see Figure $7 b$ ). The results for defect rate trend was used to verify the structure/correctness of the model to achieve the study objectives. With the knowledge that gains in defect rate can be 
achieved, experiment 3 was carried forward as a base improvement to compare with other experiments that would test the effects of altering the scheduling format.
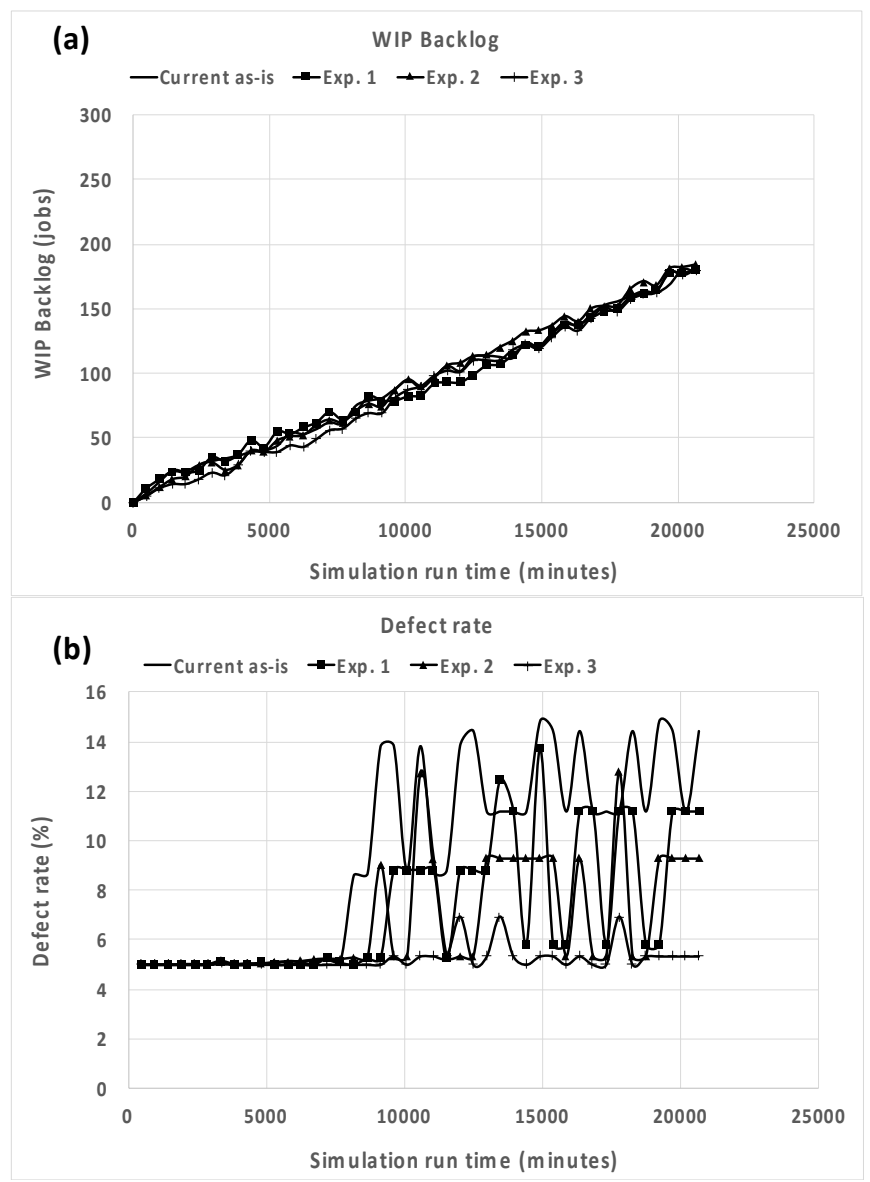

Figure 7. Simulation results to validate the SD-DES hybrid simulation model and establish the effect of cutting corners and increasing machine speed: a) trend in WIP backlog; b) trend in defect rate

Using experiment 3 as the basis for further experiments, three scheduling policies were tested on the model:

- Experiment 3a- No corners cut, no machine speed increases, limit the number of complex jobs to no more than one at a time. Currently there is no scheduling rule to limit the number of complex jobs that are scheduled to print at the same time. Complex jobs enter on a first-in-first-out basis and up to four complex jobs are sometimes printed simultaneously.

- Experiment 3b- No corners cut, no machine speed increases, limit the number of complex jobs to no more than two at a time.

- Experiment 3c- No corners cut, no machine speed increases, limit the number of complex jobs to no more than two at a time and also limit the number of simple jobs to no more than three at a time. This experiment is intended to limit the number of complex jobs to be at a pre-set level that avoids it building up or affecting quality performance. 
The simulation results for the experiments to test different scheduling policies are presented in Figure 8. From the simulation results, Exp. $3 \mathrm{c}$ gives the best result for WIP backlog trend and defect rate. The results show that in the current system, it is possible not to cut corners or increase machine speed and still achieve gains in production.
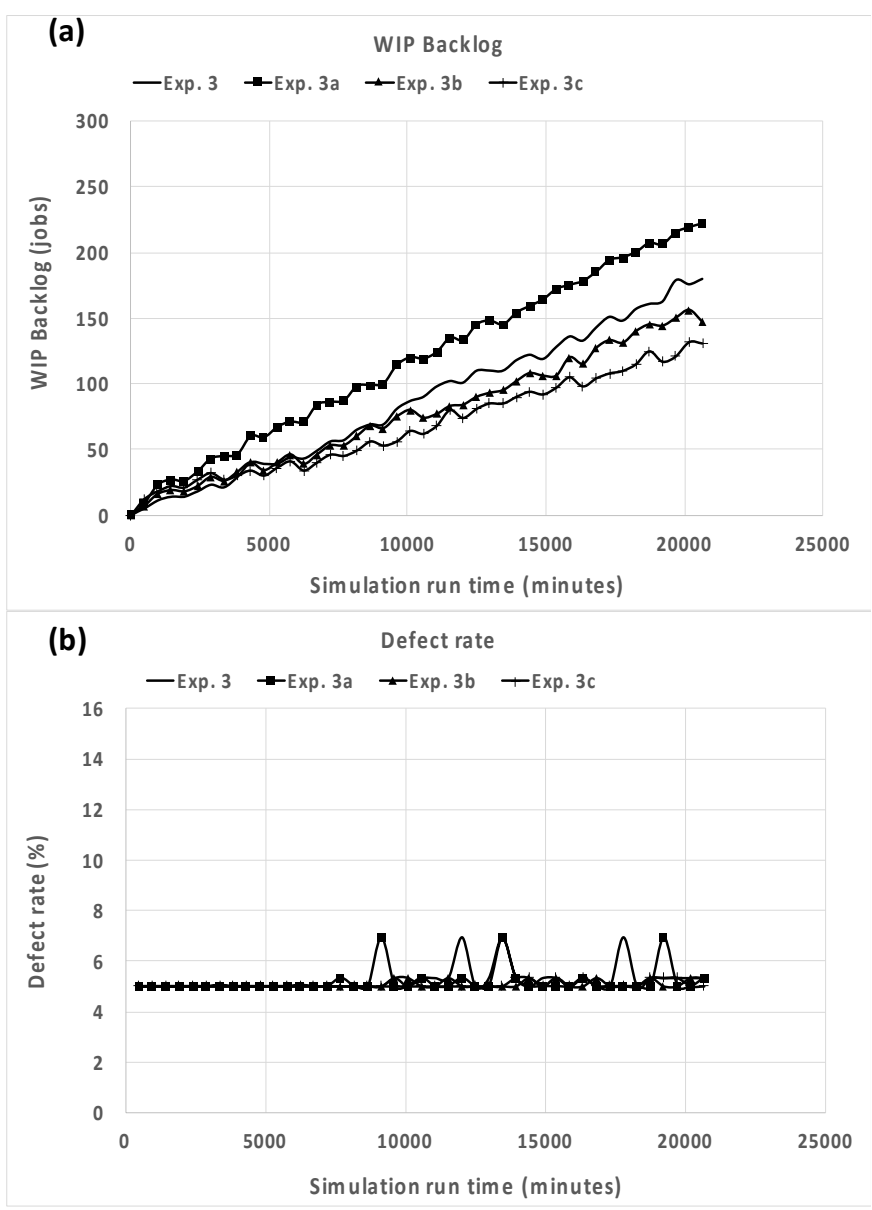

Figure 8. Simulation results to establish effect of different scheduling rules: a) trend in WIP backlog; b) trend in defect rate

\section{Discussions}

How else would the problem have been investigated? We have established that a SD-only model would be complex to code if the detailed scheduling part is to be included, otherwise many assumptions would need to be made, rendering the simulation model less realistic. It is also possible to use a DES-only model, and then assume that defect rate is a constant that varies in proportion to the number of complex jobs in the system. This may be able to address the scheduling performance but would fail to tell us why defect rate varies when number of complex jobs in the system also varies. By combining SD and DES in a hybrid fashion, the problem could be investigated in a more detail way than if the simulation methods were used as stand-alone tools. Another alternative would have been to discard either the feedback structures or the process flow, so that only one modelling method is used. However, the comprehensiveness of the simulation study and the results thereof would be doubtful since some important variables would have been excluded. 
What types of LM problems would be candidates for the hybrid SM concept? Firstly, the problem to be addressed is fit for SM, since not all problems require a simulation model to be built. Secondly, the problem requires modelling the process flow. Ensuring the product is continuously flowing in the system is one of the tenets of LM. In addition, minimizing manufacturing lead time is uppermost in the minds of manufacturing managers. Process flow and minimizing manufacturing lead time is majorly modelled in DES (Jeon and Kim, 2016). Where it is modelled using SD, it is often a high-level analysis (Ali and Deif, 2014; Sterman, 2000). Thirdly, the problem involves modelling the interaction between the production process and human factors. Previous studies have shown that the production process and Human Resource Management practices cause lean to fail or succeed in manufacturing systems (Worley and Doolen, 2015; Desai and Prajapati, 2017). Most, if not all, manufacturing systems have human beings, whose behaviours, decisions, attitudes, commitment, mindset, motives as well as (in)actions affect and are affected by the system they operate in. In other words, there is frequent, sometimes continuous human/process flow interaction going on in the system. We believe this human/production process interaction to be the main determinant of where and when to use the hybrid modelling concept, and specifically where the production process needs to be modelled in detail.

The hybrid modelling concept investigates LM systems on the basis of people and systems, as well as the decisions people take because of and resulting in changes in the system. In simulationbased literature relating to LM systems, we did not come across any study where the people aspects, such as their decision, motives and actions were included in a simulation model. It would appear that this is an area being avoided, possibly because it may seem imprecise to model such intangible aspects of the system; whereas they are real and part of the actual system being modelled (Neumann and Medbo, 2009; Baines et al., 2005; Cummings and Clare, 2015). If the human factor is not modelled, it is assumed to either be a constant or taken to be unimportant in the problem situation. Meanwhile, the hybrid SM concept advanced in the current paper is one step towards designing a toolset that will enable one establish a credible link between non-tangible as well as human resource aspects of lean and the performance of an organization. Aspects such as managers commitment, workforce adherence and employee attitude towards lean; job rotation; training, and many others which are often appraised using subjective-based instruments, can now be objectively assessed and comprehensively investigated using the hybrid modelling concept advanced in the current article.

The depth and breadth of information that the modelling concept is able to generate makes it an effective Operations Research (OR) methodology for both academicians and practitioners. The studied case is an example, where an age-old problem is presented and where managers are faced with advancing production output ahead of continuous improvement, or even advancing one improvement over another (Sarkar et al., 2011). Time spent on continuous improvement programmes for example training and Quality Circles, may be time lost in production (Repenning and Sterman, 2002; Da Silveira and Slack, 2001; Singh et al., 2013). Lean and continuous improvement could even be seen as being stressful (Conti et al., 2006) or unnecessary. This was evident in the studied case. In other situations, quality checks may even cause machines to be idle (Roth and Franchetti, 2010). Such behaviours can easily act as a barrier to lean (Salonitis and Tsinopoulos, 2016; Lodgaard et al., 2016). Lean itself may lose steam and this is one subtle way through which it slowly fails (Singh and Singh, 2012). Without the simulation-based study the managers had only partial understanding of the problem, believing it was something they had control over. Colledani et al. (2014) have asked about which tools can be used to support the joint consideration of quality and production output. We believe that the hybrid modelling concept documented in the current paper answers this question. 
The plant managers adopted the recommendations that resulted from the simulation-based study. Actual gains in the system were not far from those revealed from experimenting with the model. The study has motivated managers in the plant to use the same approach to address similar issues relating to plant maintenance. The simulation-based study was also able to convince managers that cutting on continuous improvement programmes to advance production does not yield the desired gains in production output. Prior to the simulation based study, managers in the plant had considered increasing the workforce, aware that with more machine operators the ideal quality checks can be sustained when workload in the plant increases. The results of the simulation based study was able to show that additional workers were not needed, saving the company money on labour cost.

Majority of the literature on scheduling in job shops and make to order environments such has been focused on finding an optimal schedule that would minimize tardiness and reduce manufacturing lead time. Using the hybrid SM concept on the case presented in the current study, we found that scheduling can also affect quality performance. Managers may demand full compliance to quality checks, machine operators may undergo training and standard operating procedures may be produced. All these may be in place, yet quality performance could still be diminished because the root cause (for example scheduling performance) has not been improved. Although managers are mentally aware of the connection between cause and effect variables, such as scheduling and quality issues, without a simulation model they may not have realised how to address the problem. The combination of SD and DES in a hybrid fashion enabled us to study how quality performance may be dependent on schedule performance. In a system where scheduling is critical and quality checks are done manually, if schedule performance is low, quality performance may also be low, as a result of fluctuating workload per worker. This is based on the notion that a poorly scheduled work flow will lead to increased workload and stress, which may cause workers to cut corners. While a survey-based study needs to establish this hypothesis, taking this hypothetical view will help managers focus on improvements that lessen the stress level of workers.

Although the current study environment is a make-to-order setting, the schedule-quality relationship holds for other manufacturing system types such as continuous processes and assembly lines. For example, minimizing machine downtime by reducing the frequency of shut down TPM activities because the factory is falling behind on orders, may cause quality issues to rise as machines breakdown more frequently due to lack of maintenance. This type of problem situation can be modelled using the hybrid SM concept, as it has to do with decisions managers take because the system is not coping well with factory orders, whereas the decision will eventually create further backlog in the system. The DES part of the hybrid can be used to establish an optimal setting of the maintenance function, for example.

Hybrid models of the continuous-discrete event simulation type have been in existence since the late 70s (Cellier, 1979; Coyle and Wolsterholm, 1980), and much has been documented ever since on SD-DES (or DES-SD) hybrid models. We found our approach to be a straightforward one that can be replicated in other instances. In fact, one key advantage of the hybrid simulation modelling concept is that it has been designed to be easily understood by the novice modeller, while being robust for use by the expert modeller. For example, we did not know, prior to building the first conceptual model (Figure 3) whether a hybrid was going to be applicable. How we intended to use simulation modelling approach to address the presented problem warranted the use of the SD-DES hybrid model. Others would take a different approach, presenting first an architype situation that justifies the use of SD-DES hybrid modelling. We believe our approach is unique in this regard because it is flexible and not limited to any architype situation. Another unique feature of our concept is that it commences from using a CLD and SFD, whereas others would commence the hybrid formation with a parallel build of both the SD and DES. This would 
likely be the case if one has prior knowledge that he/she would be using SD-DES hybrid SM. Starting the SM process with a CLD ensures that all the key variables relating to the problem situation are captured as well as the important feedback structures and interactions that define how people and systems interact in real life. Building the SFD next also enables one establish what will be modelled using DES, what will be modelled using SD and how the sub-models would communicate. We believe all these procedures makes our approach simple and adaptable. We also believe that our approach will enable the set of procedures in SD-DES hybrid SM based work to be formalized. As it is now, it may appear confusing to the new comer in SM when presented with the plethora of SD-DES hybrid frameworks that have been advanced. Fortunately, there are now mixed-method SM toolsets such as AnyLogic and ExtendSim that enable the simplification of data exchange mechanisms in hybrid modelling, which in the past was a complex undertaking.

The problem that was presented in the current study was obviously a candidate for SD-DES hybrid SM. SD-DES hybrid simulation models have been advanced in Health Care, Construction and even in Manufacturing systems. We did not come across the use of a SD-DES hybrid simulation model in LM systems. So, even if we wanted to apply a SD-DES hybrid SM concept to the current case, there were no foundational studies we could build on. With the current study, this gap has been bridged. We believe that the step-by-step process in the current simulationbased study has been explicitly documented. It is intended that the reports of multiple case studies such as the one presented here will be chronicled and used to formalize guidelines for the use of a generalized SD-DES hybrid SM concept, since it appears that there are different types of SDDES hybrid frameworks being advanced.

\section{Conclusion and future outlook}

The current work is motivated by the dearth of simulation modelling methods to assist lean managers understand deeply the variables and concepts that may derail lean in their systems. A system dynamics-discrete event hybrid simulation modelling concept was developed to assist managers in a lean manufacturing system decide how best to overcome such challenges. The hybrid modelling concept investigates lean manufacturing systems on the basis of people and systems, as well as the decisions people take because of and resulting in changes in the system.

The current study set out to address issues relating to Quality Management, whereas Employee Attitude towards lean as well as Scheduling needed to be included in the overall investigation. In a way, three lean concepts were investigated. There are other variables relating to Quality Management aside quality checks such as defective machine and defective raw materials. There are also other lean manufacturing practices and concepts such as Kanban, CONWIP, 5S, SMED, TPM, Single piece flow which can be investigated using the hybrid modelling concept advanced in the current research. SD-DES hybrid SM concept in LM systems is still a virgin area and there is ample opportunity for its application. The hybrid modelling concept advanced in the current research no doubt lays a foundation for this.

Time did not permit us to model issues relating to a longer term. It would be interesting to use the hybrid modelling concept to investigate a long-term problem situation for a LM system. One that the present authors are currently working on involves the long-term effect of skipping TPM activities because production output is lagging targets. 


\section{References}

Abduaziz O, Cheng JK, Tahar RM, et al. (2015) A Hybrid Simulation Model for Green Logistics Assessment in Automotive Industry. Procedia Engineering 100: 960-969.

Ali RM and Deif AM. (2014) Dynamic Lean Assessment for Takt Time Implementation. Procedia CIRP 17: 577-581.

Alvanchi A, Lee S and AbouRizk S. (2011) Modeling Framework and Architecture of Hybrid System Dynamics and Discrete Event Simulation for Construction. Computer-Aided Civil and Infrastructure Engineering 26: 77-91.

Alzraiee H, Zayed T and Moselhi O. (2012) Methodology for synchronizing Discrete Event Simulation and System Dynamics models. Proceedings Title: Proceedings of the 2012 Winter Simulation Conference (WSC). 1-11.

Baines TS, Asch R, Hadfield L, et al. (2005) Towards a theoretical framework for human performance modelling within manufacturing systems design. Simulation Modelling Practice and Theory 13: 486-504.

Balci O. (1998) Verification, validation, and testing. Handbook of simulation 10: 335-393.

Barbosa C and Azevedo A. (2017) Hybrid simulation for complex manufacturing value-chain environments. Procedia Manufacturing 11: 1404-1412.

Barton PI. (2000) Modeling, simulation and sensitivity analysis of hybrid systems. International Symposium In Computer-Aided Control System Design: 117-122.

Borshchev A, Karpov Y and Kharitonov V. (2002) Distributed simulation of hybrid systems with AnyLogic and HLA. Future Generation Computer Systems 18: 829-839.

Brailsford SC, Desai SM and Viana J. (2010) Towards the holy grail: Combining system dynamics and discrete-event simulation in healthcare. . Proceedings of the 2010 Winter Simulation Conference. 2293-2303.

Cellier FE. (1979) Combined continuous/discrete system simulation by use of digital computers: techniques and tools. Swiss Federal Institute of Technology, Zurich.

Chahal K, Mustafee N, Eldabi T, et al. (2013) A conceptual framework for hybrid system dynamics and discrete event simulation for healthcare. Journal of Enterprise Information Management 26: 50-74.

Chatha K and Weston R. (2006) Combined discrete event simulation and systems thinking-based framework for management decision support. Proceedings of the Institution of Mechanical Engineers, Part B: Journal of Engineering Manufacture 220: 1969-1981.

Chongwatpol J and Sharda R. (2013) Achieving Lean Objectives through RFID: A SimulationBased Assessment. Decision sciences 44: 239-266.

Colledani M, Tolio T, Fischer A, et al. (2014) Design and management of manufacturing systems for production quality. CIRP Annals-Manufacturing Technology 63: 773-796.

Conti R, Angelis J, Cooper C, et al. (2006) The effects of lean production on worker job stress. International Journal of Operations \& Production Management 26: 1013-1038. 
Coyle RG and Wolsterholm EP. (1980) Modelling discrete events in System Dynamics model. A case study. Dynamica 6: 21-27.

Cummings M and Clare A. (2015) Holistic modelling for human-autonomous system interaction. Theoretical Issues in Ergonomics Science 16: 214-231.

Da Silveira G and Slack N. (2001) Exploring the trade-off concept. International Journal of Operations \& Production Management 21: 949-964.

Desai D and Prajapati BN. (2017) Competitive advantage through Six Sigma at plastic injection molded parts manufacturing unit: A case study. International Journal of Lean Six Sigma 8: 411-435.

Dierks MM, Dulac N and Leveson NG. (2008) System Dynamics Approach to Model Risk in Complex Healthcare Settings/ Time Constraints. Production Pressures and Compliance with Safety Controls.

Djanatliev A and German R. (2013) Prospective healthcare decision-making by combined system dynamics, discrete-event and agent-based simulation. Proceedings of the 2013 Winter Simulation Conference: Simulation: Making Decisions in a Complex World. IEEE Press, 270-281.

Donzelli P and Iazeolla G. (2001) A Hybrid Software Process Simulation Model. Software Process. Improvement and Practice 6: 97-109.

Eldabi T, Balaban M, Brailsford S, et al. (2016) Hybrid simulation: Historical lessons, present challenges and futures. Winter Simulation Conference (WSC), 2016. IEEE, 1388-1403.

Goodson RE. (2002) Read a plant-fast. Harvard business review 80: 105-113.

Greasley A. (2005) Using system dynamics in a discrete-event simulation study of a manufacturing plant. International Journal of Operations \& Production Management 25: 534-548.

Green JJ, Krejci C and Cantor DE. (2017) A Hybrid Simulation Model of Helping Behavior. Proceedings of the 2017 Winter Simulation Conference. Las Vegas.

Hadid W, Mansouri SA and Gallear D. (2016) Is lean service promising? A socio-technical perspective. International Journal of Operations \& Production Management 36: 618642.

Hao Q and Shen W. (2008) Implementing a hybrid simulation model for a Kanban-based material handling system. Robot. Comput. Integr. Manuf 24: 635-646.

Helal M. (2008) A hybrid system dynamics-discrete event simulation approach to simulating the manufacturing enterprise: University of Central Florida.

Inam A, Adamowski J, Halbe J, et al. (2015) Using causal loop diagrams for the initialization of stakeholder engagement in soil salinity management in agricultural watersheds in developing countries: A case study in the Rechna Doab watershed, Pakistan. Journal of environmental management 152: 251-267.

Jahangirian M, Eldabi T, Naseer A, et al. (2010) Simulation in manufacturing and business: A review. European Journal of Operational Research 203: 1-13.

Jeon SM and Kim G. (2016) A survey of simulation modeling techniques in production planning and control (PPC). Production Planning \& Control 27: 360-377. 
Lättilä L, Hilletofth P and Lin B. (2010) Hybrid simulation models - When, Why, How? Expert Systems with Applications 37: 7969-7975.

Law AM and Kelton WD. (1991) Simulation modeling and analysis: McGraw-Hill New York.

Lee YH, Cho MK, Kim SJ, et al. (2002) Supply chain simulation with discrete-continuous combined modeling. Computers \& Industrial Engineering 43: 375-392.

Liker JK. (2004) The toyota way: 14 Management Principles from the World's Greatest Manufacturer, New York: McGraw-Hill.

Lodgaard E, Ingvaldsen JA, Gamme I, et al. (2016) Barriers to Lean Implementation: Perceptions of Top Managers, Middle Managers and Workers. Procedia CIRP 57: 595-600.

Morgan JS, Howick S and Belton V. (2017) A toolkit of designs for mixing Discrete Event Simulation and System Dynamics. European Journal of Operational Research 257: 907918.

Neumann W and Medbo P. (2009) Integrating human factors into discrete event simulations of parallel flow strategies. Production Planning and Control 20: 3-16.

Oleghe O and Salonitis K. (2015) Improving the Efficacy of the Lean Index through the Quantification of Qualitative Lean Metrics. Procedia CIRP 37: 42-47.

Oliveira M, Montevechi J, Pinho A, et al. (2017) Using hybrid simulation to represent the human factor in production systems. International Journal of Simulation Modelling (IJSIMM) 16.

Omogbai O and Salonitis K. (2016) Manufacturing System Lean Improvement Design Using Discrete Event Simulation. Procedia CIRP 57: 195-200.

Owens PL. (2006) One more reason not to cut your training budget: The relationship between training and organizational outcomes. Personnel Administration 35: 163-172.

Probst TM and Graso M. (2013) Pressure to produce= pressure to reduce accident reporting? Accident Analysis \& Prevention 59: 580-587.

Pruckner M and German R. (2013) A hybrid simulation model for large-scaled electricity generation systems. Proceedings of the 2013 Winter Simulation Conference: Simulation: Making Decisions in a Complex World. IEEE Press, 1881-1892.

Rabelo L, Helal M, Jones A, et al. (2005) Enterprise simulation: a hybrid system approach. International Journal of Computer Integrated Manufacturing 18: 498-508.

Rabelo L, Sarmiento AT, Helal M, et al. (2015) Supply chain and hybrid simulation in the hierarchical enterprise. International Journal of Computer Integrated Manufacturing 28: 488-500.

Repenning NP and Sterman JD. (2002) Capability traps and self-confirming attribution errors in the dynamics of process improvement. Administrative Science Quarterly 47: 265-295.

Robinson S. (2015) A tutorial on conceptual modeling for simulation. Proceedings of the 2015 Winter Simulation Conference. IEEE Press, 1820-1834.

Robinson S, Brooks R, Kotiadis K, et al. (2010) Conceptual modeling for discrete-event simulation, New York: CRC Press. 
Robinson S, Radnor ZJ, Burgess N, et al. (2012) SimLean: Utilising simulation in the implementation of lean in healthcare. European Journal of Operational Research 219: 188-197.

Roth N and Franchetti M. (2010) Process improvement for printing operations through the DMAIC Lean Six Sigma approach: a case study from Northwest Ohio, USA. International Journal of Lean Six Sigma 1: 119-133.

Salonitis K and Tsinopoulos C. (2016) Drivers and Barriers of Lean Implementation in the Greek Manufacturing Sector. Procedia CIRP 57: 189-194.

Sarkar A, Ranjan Mukhopadhyay A and Ghosh SK. (2011) Selection of critical processes for "process improvement". International Journal of Lean Six Sigma 2: 356-370.

Singh J and Singh H. (2012) Continuous improvement approach: state-of-art review and future implications. International Journal of Lean Six Sigma 3: 88-111.

Singh R, Gohil AM, Shah DB, et al. (2013) Total productive maintenance (TPM) implementation in a machine shop: A case study. Procedia Engineering 51: 592-599.

Sterman J. (2000) Business dynamics: systems thinking and modeling for a complex world, Boston, USA: Mc Graw-Hill.

Sung SY and Choi JN. (2014) Do organizations spend wisely on employees? Effects of training and development investments on learning and innovation in organizations. Journal of organizational behavior 35: 393-412.

Tejada JJ, Ivy JS, King RE, et al. (2014) Combined DES/SD model of breast cancer screening for older women, II: screening-and-treatment simulation. IIE Transactions 46: 707-727.

Tekippe AJ and Krejci CC. (2016) Using hybrid simulation modeling to assess the dynamics of compassion fatigue in veterinarian general practitioners. Winter Simulation Conference (WSC), 2016. IEEE, 1352-1363.

Venkateswaran J and Son YJ. (2005) Hybrid system dynamic - discrete event simulation-based architecture for hierarchical production planning. International Journal of Production Research 43: 4397-4429.

Womack JP, Jones DT and Roos D. (1990) Machine that changed the world: Simon and Schuster.

Woods DD. (2017) Essential characteristics of resilience. Resilience engineering. CRC Press, 3346.

Worley JM and Doolen TL. (2015) Organizational structure, employee problem solving, and lean implementation. International Journal of Lean Six Sigma 6: 39-58.

Yin RK. (2014) Case study research: Design and methods: Sage publications.

Zulkepli J, Eldabi T and Mustafee N. (2012) Hybrid simulation for modelling large systems: An example of integrated care model. Proceedings of the 2012 Winter Simulation Conference. 1-12. 
2018-12-31

Hybrid simulation modelling of the human-production process interface in lean manufacturing systems

\section{Oleghe, Omogbai}

Emerald

Omogbai Oleghe and Konstantinos Salonitis. (2018) Hybrid simulation modelling of the human-production process interface in lean manufacturing systems. International Journal of Lean Six Sigma, Volume 10, Issue 2, 2019, pp. 665-690

https://doi.org/10.1108/lJLSS-01-2018-0004

Downloaded from Cranfield Library Services E-Repository 